\title{
Polypharmacy: a risk to the health of the elderly
}

\section{Introduction}

Polypharmacy defined as the use of several drugs simultaneously is one of the main risk factors for the occurrence of drug-related problems. The risk of occurrence increases with the use of two drugs, quadrupling when this number increases to five, and increases up to eight times in cases where seven or more drugs are consumed. ${ }^{1-2}$

Polypharmacy has increased in the last decades and will continue to increase as populations age and the number of people with multiple long-term conditions increases. However, it exposes the elderly to drug interactions and adverse drug reactions. There are flaws in the evaluation of which drugs may not be beneficial and therefore discontinued..$^{2-3}$

In an Indian study, Jain et al. analyzed potential theoretical drug interactions in patients hospitalized in a cardiology unit and identified $70 \%$ of the interactions classified as higher intensity, where most involved antiplatelet drugs, and found a positive correlation between the number of drugs prescribed and the frequency of potential drug interactions. ${ }^{4}$

In the Venezuelan cross-sectional, descriptive and observational study, Lo Presti et al. investigated the theoretical drug interactions in hospitalized patients with cardiovascular diseases and found that $85 \%$ of the identified interactions had a general pharmacokinetic mechanism involving the biotransformation stage, and the other part was related to the pharmacodynamic process, synergism. Most of the interactions involved antiplatelet, anticoagulant and anti-lipemic. The researchers concluded that the number of potential drug interactions increases with the number of drugs prescribed. ${ }^{5}$

In the Swedish study, conducted by clinical pharmacists between the years 2015 and 2016, Peterson et al. investigated the prevalence and types of drug-related problems and associated factors in patients hospitalized in a teaching hospital. Clinical pharmacists have identified $66 \%$ of drug-related problems, including inadequate use and drug interactions involving mainly drugs that play a role in the cardiovascular system. ${ }^{6}$

In an English study, Anderson and Lakhani evaluated the collaborative work between physicians and pharmacists in the elderly exposed to polypharmacy highlights the potential benefits of the joint work of these professionals starting in the last years of graduation in the identification of inconsistent prescriptions and drug interactions that require greater professional interaction. ${ }^{7}$

In the German study conducted in a teaching hospital, Lenssen et al. verified the pharmacist's performance in medication reconciliation, safety analysis of pharmacotherapy, and drug-related problems. The study identified 2.3 drug-related problems per patient, mainly associated with drug numbers and age, and drug interactions accounted for $34 \%{ }^{8}$

In a Brazilian study, conducted between 2009 and 2010, Santos et al. evaluated the potential drug interactions among medications used by the elderly. The authors concluded that most of the potential interactions were related to routinely used medications and more than one third of the elderly were exposed to the larger interactions. Drugs
Volume 3 Issue I - 2018

\section{Tiago Aparecido Maschio de Lima}

Clinical Research Coordinator, Centro Integrado de Pesquisa, Fundação Faculdade Regional de Medicina de São José do Rio Preto, Brazil

Correspondence: Tiago Aparecido Maschio de Lima, Clinical Research Coordinator, Centro Integrado de Pesquisa, Fundação Faculdade Regional de Medicina de São José do Rio Preto, Brazil, Tel 55I7320I-5054, Fax 55-17-320I-5I54,

Email tiagomaschio.farmacip@gmail.com

Received: August 14, 2017 | Published: January 22, 2018

with a narrow therapeutic index were involved in several interactions, with a higher risk of toxicity. The recommended clinical management procedures were dose adjustment and dosage changes, control of serum drug levels and monitoring of clinical conditions. ${ }^{9}$

Still in Brazil, in a cross-sectional study, Figueiredo et al. analyzed the occurrence of adverse drug reactions during the hospitalization of the elderly in a teaching hospital. The study demonstrated a high prevalence of adverse drug reactions among the elderly and a positive association with hospitalization time. The most frequent adverse drug reactions were acute renal damage, hypotension and skin reactions. In addition, it detected a positive association between drug-drug interactions and hospitalization for diagnosis of circulatory disease and number of medications greater than $14 .{ }^{10}$

In another Brazilian study, Giordani et al. performed a documentary analysis of adverse reactions in hospitalized patients in a teaching hospital, through retrospective review of medical records using as traceability criteria as an instrumental tool. The researchers concluded that $1 / 6$ of the hospitalized patients presented adverse drug events, the amount of medication prescribed and the length of hospitalization favored the occurrence of the events. ${ }^{11}$

\section{Conclusion}

despite advances in Evidence-Based Health, it is necessary new researchs on the impact of interventions on elderly with polypharmacy, mainly about the technological solutions and and the results of interventions by different health professionals. For now, it is possible to highlight the importance of prescription optimization in reducing the risks caused by the polypharmacy in the elderly, such as the drug-drug interactions, the adverse reactions and the intoxications by medicines, through the integrated action between Pharmacists, Physicians and Nurses.

\section{Acknowledgements}

None.

\section{Conflict of interest}

Author declare that there is no conflict of interest. 


\section{References}

1. Molokhia M, Majeed A. Current and future perspectives on the management of polypharmacy. BMC Fam Pract. 2017;18(1):70.

2. Schöttker B, Saum K, Muhlack DC, et al. Polypharmacy and mortality: new insights from a large cohort of older adults by detection of effect modification by multi-morbidity and comprehensive correction of confounding by indication. Eur JClin Pharmacol.2017;73(8):1041-1048.

3. Abolbashari M, Macaulay TE, Whayne TF, et al. Polypharmacy in Cardiovascular Medicine: Problems and Promises! Cardiovasc Hematol Agents Med Chem. 2017;15(1):31-39.

4. Jain S, Jain P, Sharma K, et al. A Prospective Analysis of Drug Interactions in Patients of Intensive Cardiac Care Unit. J Clin Diagn Res. 2007;11(3):FC01-FC04.

5. Lo Presti A, Fernández Y, Carrillo M, et al. Potential pharmacological interactions in patients with polymedicated cardiovascular disease. Salus. 2015;19(2):11-17.
6. Peterson C, Gustafsson M. Characterisation of Drug-Related Problems and Associated Factors at a Clinical Pharmacist Service-Naïve Hospital in Northern Sweden. Drugs Real World Outcomes. 2017;4(2):97-107.

7. Anderson E, Lakhani N. Interprofessional learning on polypharmacy. Clin Teach. 2016;13(4):291-297.

8. Lenssen R, Heidenreich A, Schulz JB, et al. Analysis of drug-related problems in three departments of a German University hospital. Int $J$ Clin Pharm. 2016;38(1):119-126.

9. Santos TRA, Silveira EA, Pereira LV, et al. Potential drug-drug interactions in older adults: A population-based study. Geriatr Gerontol Int. 2017;17(12):2336-2346.

10. Figueiredo TP, Souza GRC, Barroso SCC, et al. Factors associated with adverse drug reactions in older inpatients in teaching hospital. Int J Clin Pharm. 2017;39(4):679-685.

11. Giordani F, Rozenfeld S, Martins M. Adverse drug events identified by triggers at a teaching hospital in Brazil. BMC Pharmacol Toxicol. 2014;15:71. 